\title{
On the Velocity Gradient in Stably Stratified Sheared Flows. Part 2: Observations and Models
}

\author{
Rostislav D. Kouznetsov • Sergej S. Zilitinkevich
}

Received: 6 October 2009 / Accepted: 9 March 2010 / Published online: 28 March 2010

(C) The Author(s) 2010. This article is published with open access at Springerlink.com

\begin{abstract}
Observations of the dependence of the dimensionless wind speed gradient $\phi_{m}$ as a function of the Monin-Obukhov stability parameter $z / L_{o}$ under strong stability diverge from results of large-eddy simulation (LES) modelling. A kinetic energy budget analysis indicates that it is likely caused by violations of the assumptions of stationarity and/or homogeneity of turbulence in the field experiments rather than in imperfections of the LES. This confirms the validity of the widely used linear approximation for $\phi_{m}$ not only at weak to moderate stability, but also under strong stability. The new interpretation of the linear approximation of $\phi_{m}$ is given in terms of turbulent scales, which gives hope for its applicability to the free atmosphere as well.
\end{abstract}

Keywords Flux-profile relationships - Stable atmospheric boundary layer .

Turbulent length scale

\section{Introduction}

Recently Zilitinkevich et al. (2010) have formulated a new theoretical derivation of the well-known expression for the dimensionless wind speed gradient $\phi_{m}$ as a function of Monin-Obukhov stability parameter $z / L_{o}$, and concluded that it is a physical law rather

R. D. Kouznetsov $(\varangle) \cdot$ S. S. Zilitinkevich

Finnish Meteorological Institute, P.O. Box 503, 00101 Helsinki, Finland

e-mail: rostislav.kouznetsov@fmi.fi

R. D. Kouznetsov · S. S. Zilitinkevich

Obukhov Institute of Atmospheric Physics, Moscow, Russia

S. S. Zilitinkevich

Division of Atmospheric Sciences, University of Helsinki, Helsinki, Finland

S. S. Zilitinkevich

Nansen Environmental and Remote Sensing Centre/Bjerknes Centre for Climate Research,

University of Bergen, Bergen, Norway 
than an approximation of empirical data. However, the results of most known natural experiments do not reproduce such a behaviour of $\phi_{m}$. In the present study we show that the deviations are caused by a violation in the assumptions of homogeneity and/or stationarity in the experiments rather than problems in the expression itself.

The similarity theory of the thermally stratified atmospheric surface layer states that any properly scaled dimensionless parameter of turbulence is a unique function of the dimensionless height. In particular, the wind shear

$$
\frac{\partial U}{\partial z} \frac{\kappa z}{u_{*}}=\phi_{m}\left(z / L_{o}\right),
$$

where $U$ is the mean horizontal wind speed, $z$ is the height above the surface, $\kappa=0.4$ is the von Kármán constant, $u_{*}$ is friction velocity $=\sqrt{-\overline{u w}}, \overline{w \theta}$ is the temperature flux, $g$ is the acceleration due to gravity, $\beta$ is the buoyancy parameter $=g / T_{0}\left(T_{0}\right.$ is reference temperature), $L_{o}$ is the Obukhov length scale $=-u_{*}^{3} /(\beta \kappa \overline{w \theta})$. The similarity theory does not state the explicit form of the $\phi_{m}$ function, so it is to be determined experimentally.

The most widely-used empirical expression for $\phi_{m}\left(z / L_{o}\right)$ was suggested half a century ago (McVehil 1964), as

$$
\phi_{m}\left(z / L_{o}\right)=1+\alpha \frac{z}{L_{o}},
$$

where a value of $\alpha=5$ is commonly used (Dyer 1974). (Note, that hereafter $\kappa$ is included into the definition of $L_{o}$, unlike in Zilitinkevich et al. (2010) ${ }^{1}$.)

Zilitinkevich et al. (2010) derived Eq. 2 from the concept of the critical flux Richardson number. Earlier, the same expression was derived from the $z$-less concept. However, the $z$-less concept leads to unrealistic expressions for some other parameters, in particular the potential temperature gradient (see Zilitinkevich et al. 2010).

The expression (2) has been verified by natural (Businger et al. 1971; Högström 1988) and numerical (Basu and Porté-Agel 2006) experiments under relatively weak stratification $z / L_{o} \lesssim 1$. Theoretical considerations and large-eddy simulations (LES) by Zilitinkevich and Esau (2007) suggest that the Eq. 2 is valid within the whole boundary layer if one uses the local $z$-dependent values of the Obukhov length and friction velocity. However, under strong stratification results of different experimental studies are contradictory. LES simulations (Zilitinkevich and Esau 2007; Basu and Porté-Agel 2006) and some field measurements (Hicks 1976) confirm the expression (2); other field experiments suggest a slower than linear increase of $\phi_{m}$ at $z / L_{o} \gg 1$ (Yagüe et al. 2006; Grachev et al. 2007) or even its levelling off (Cheng and Brutsaert 2005).

\section{Test Against $R f_{c}$}

Natural experiments are difficult from a methodological point of view, since atmospheric turbulence cannot be controlled and even monitored well. On other hand, such experiments are very important for understanding the applicability of specific theoretical considerations for real atmospheric processes. This justifies the importance of finding a consensus between natural and numerical experiments.

To illustrate the discrepancies consider the results of LES modelling (Esau and Zilitinkevich 2006) and of the observations from the SHEBA (Surface HEat Budget of the Arctic Ocean) field experiment (Grachev et al. 2007). The plots of the dimensionless wind

1 Such a form is traditionally used in experimental studies. Thus in this paper the symbol $L_{o}$ is used. 

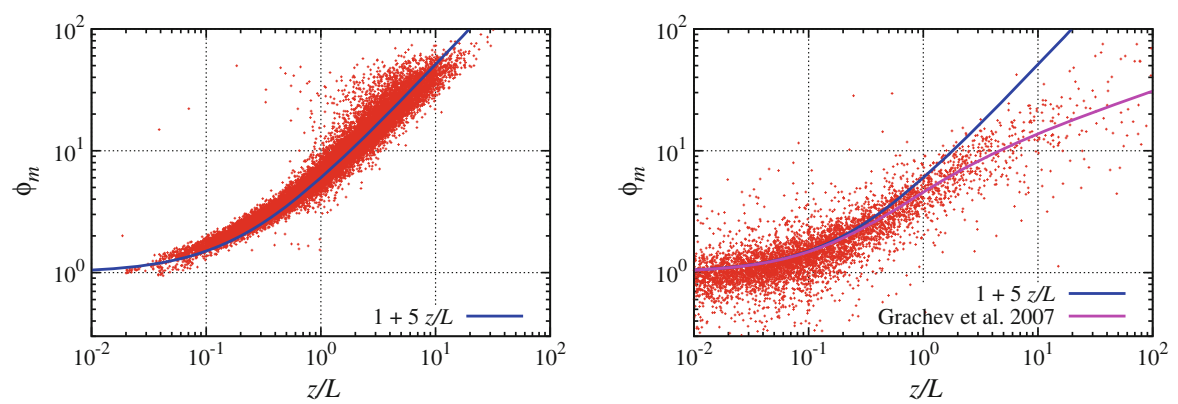

Fig. 1 The dimensionless wind speed gradient as a function of dimensionless height $z / L_{o}$. Points are calculated from LES DATABASE64 (left) and from SHEBA experimental data (right)

Fig. 2 The scatter plot of the shear production term of TKE budget versus buoyancy term calculated from SHEBA data. Lines correspond to different values of $R f$



shear (Eq. 1) for these datasets are shown in Fig. 1. Observations from the other above-mentioned field experiments indicate a behaviour in $\phi_{m}$ similar to that for SHEBA data.

Under very stable stratification $\left(z \gg L_{o}\right)$, the expressions (2) and (1) give

$$
-\overline{u w} \frac{d U}{d z}=\alpha \beta \overline{w \theta}
$$

which suggests that a certain fraction of shear production of turbulent kinetic energy (TKE) is consumed by buoyancy. The data points on shear production and buoyancy from the SHEBA experiment for $z / L_{o}>10$ are shown in Fig. 2. Unlike Fig. 1, such a representation is free of the problems arising from self-correlation or inaccuracies caused by taking the ratio of two small numbers. The lines in the figure correspond to the values of the flux Richardson number $R f=\beta \overline{w \theta} /(\overline{u w} \partial U / \partial z)=0.2,1$, and 5 , and one can see that most of the data points in Fig. 2 have $R f>1$, i.e. buoyancy consumes more energy than wind shear produces. This can occur only if the turbulence is strongly non-stationary or inhomogeneous.

The data of other above mentioned experiments have the same problem. Since stationarity is usually checked during the data processing, the problem appears most likely to be due to strong vertical inhomogeneity in the mean flow and turbulence. The measured wind speed gradient is essentially averaged over several metres (the distance between sensors) whereas the fluxes are measured at a point. This leads to the underestimation of the dimensionless wind-speed gradient, which causes excessive Richardson flux numbers and deviations of the observations from expression (2). 


\section{Scale Interpretation of $\phi_{m}$}

The expression (2) contains the height $z$ on both sides. In flows similar to those in the free atmosphere, where the surface can be considered as being far away, the explicit dependence on $z$ should not appear. This fact makes the applicability of the expression (2) for such flows doubtful. However, the $z$-dependence in (2) can be made clearer by dividing throughout by $z$ :

$$
\frac{\partial U}{\partial z} \frac{1}{u_{*}}=\frac{1}{\kappa z}+\frac{1}{\kappa R f_{c}} \frac{1}{L_{o}},
$$

This expression shows that the shear scale (on the left) is limited by the distance to the wall and by the Obukhov length scale. Under strong stability (or large $z$ ) the first term on the right-hand side of (4) can be neglected. This expression has a similar form to those used by e.g. Zilitinkevich et al. (2007) to derive the turbulent scale from the scales of several limiting factors. Note, that Zilitinkevich et al. (2007) used the squared reciprocals, whereas (4) contains linear terms. Indeed, the exact form for accounting for the limiting factors is more or less ad hoc and has no physical basis. However, the good agreement of (2) (and, consequently, (4)) with observations and models shows the correctness of this approach.

\section{Conclusions}

The TKE budget consideration shows that any form of $\phi_{m}\left(z / L_{o}\right)$ that increases slower than $5 z / L_{o}$ under strong stability implies supercritical flux Richardson numbers and is thus inconsistent with the assumption of homogeneous stationary turbulence. Any experimental data indicating such a behaviour of $\phi_{m}\left(z / L_{o}\right)$ should be considered as violating the assumption. The inconsistency of the expression (2) with the data from natural experiments does not indicate its incorrect nature, but rather indicates a misinterpretation of the data.

The expression (2) can be interpreted in terms of scales, and gives hope that it is applicable not only in the surface layer, where the Monin-Obukhov similarity is valid, but also to free-atmosphere-like flows. Moreover, the scale interpretation gives a hint on how to account for other factors that limit the shear scale in a simpler way than was proposed by Zilitinkevich and Esau (2007). However, both these suggestions require comprehensive experimental verification.

Acknowledgements We would like to thank Prof. M.A. Kallistratova for helpful discussions, and are grateful to Dr. I. Esau from Nansen Environmental and Research Center for providing the LES database. The SHEBA data were provided by NCAR/EOL under sponsorship of the National Science Foundation (http:// data.eol.ucar.edu/). This work has been supported by the EC FP7 project ERC PBL-PMES (No. 227915) and by Russian Foundation for Basic Research (Grants 08-05-00671 and 10-05-00802).

Open Access This article is distributed under the terms of the Creative Commons Attribution Noncommercial License which permits any noncommercial use, distribution, and reproduction in any medium, provided the original author(s) and source are credited.

\section{References}

Basu S, Porté-Agel F (2006) Large-eddy simulation of stably stratified atmospheric boundary layer turbulence: a scale-dependent dynamic modeling approach. J Atmos Sci 63: 2074-2091

Businger JA, Wyngaard JC, Izumi Y, Bradley EF (1971) Flux profile relationships in the atmospheric surface layer. J Atmos Sci 28: 181-189 
Cheng Y, Brutsaert W (2005) Flux-profile relationships for wind speed and temperature in the stable atmospheric boundary layer. Boundary-layer Meteorol 114: 519-538

Dyer AJ (1974) A review of flux-profile relationships. Boundary-layer Meteorol 7: 363-372

Esau IN, Zilitinkevich SS (2006) Universal dependences between turbulent and mean flow parameters in stably and neutrally stratified planetary boundary layers. Nonlinear Proc Geophys 13: 135-144

Grachev AA, Andreas EL, Fairall CW, Guest PS, Persson POG (2007) SHEBA flux-profile relationships in the stable atmospheric boundary layer. Boundary-layer Meteorol 124: 315-333

Hicks BB (1976) Wind profile relationships from the 'Wangara' experiment. Q J Roy Meteorol Soc 102: $535-551$

Högström U (1988) Non-dimensional wind and temperature profiles in the atmospheric surface layer: a re-evaluation. Boundary-layer Meteorol 42: 55-78

McVehil GE (1964) Wind and temperature profiles near the ground in stable stratification. Q J Roy Meteorol Soc 90: 136-146

Yagüe C, Viana S, Maqueda G, Redondo JM (2006) Influence of stability on the flux-profile relationships for wind speed, $\Phi_{m}$, and temperature, $\Phi_{h}$, for the stable atmospheric boundary layer. Nonlinear Proc Geophys 13: 185-203

Zilitinkevich SS, Esau IN (2007) Similarity theory and calculation of turbulent fluxes at the surface for the stably stratified atmospheric boundary layer. Boundary-layer Meteorol 125: 193-205

Zilitinkevich SS, Elperin T, Kleeorin N, Rogachevskii I (2007) Energy- and flux-budget (EFB) turbulence closure model for stably stratified flows. Part I: steady-state, homogeneous regimes. Boundary-layer Meteorol 125: 167-191

Zilitinkevich SS, Esau I, Kleeorin N, Rogachevskii I, Kouznetsov RD (2010) On the velocity gradient in the stably stratified sheared flows. Part 1: asymptotic analysis and applications. Boundary Layer Meteorol. doi:10.1007/s10546-010-9488-X 